\title{
Chinese expert consensus on the diagnosis of osteoporosis by imaging and bone mineral density
}

\author{
Xiaoguang Cheng ${ }^{1}$, Huishu Yuan ${ }^{2}$, Jingliang Cheng ${ }^{3}$, Xisheng Weng $^{4}$, Hao Xu $^{5}$, Jianbo Gao ${ }^{6}$, \\ Mingqian Huang ${ }^{7}$, Yì Xiáng J. Wáng ${ }^{8}$, Yan Wu ${ }^{6}$, Wenjian $\mathrm{Xu}^{9}$, Li Liu ${ }^{10}$, Hua Liu ${ }^{10}$, Chen Huang ${ }^{11}$, \\ Zhengyu Jin ${ }^{12}$, Wei Tian $^{13}$; on behalf of Bone and Joint Group of Chinese Society of Radiology, Chinese \\ Medical Association (CMA), Musculoskeletal Radiology Society of Chinese Medical Doctors Association, \\ Osteoporosis Group of Chinese Orthopedic Association, Bone Density Group of Chinese Society of \\ Imaging Technology, CMA*
}

${ }^{1}$ Department of Radiology, Beijing Jishuitan Hospital, Beijing, China; ${ }^{2}$ Department of Radiology, Peking University Third Hospital, Beijing, China; ${ }^{3}$ Department of MRI, First Affiliated Hospital of Zhengzhou University, Zhengzhou, China; ${ }^{4}$ Department of Orthopedics, Peking Union Medical College Hospital, Beijing, China; ${ }^{5}$ Department of Nuclear Medicine, First Affiliated Hospital, Jinan University, Guangzhou, China; ${ }^{6}$ Department of Radiology, the First Affiliated Hospital of Zhengzhou University, Zhengzhou, China; ${ }^{7}$ Department of Radiology, Mount Sinai Hospital, New York, USA; ${ }^{8}$ Department of Imaging and Interventional Radiology, The Chinese University of Hong Kong, Hong Kong, China; ${ }^{9}$ Department of Radiology, The Affiliated hospital of Qingdao University, Qingdao, China; ${ }^{10}$ Forensic Medical Examination Center of Beijing Public Security Bureau, Beijing, China; ${ }^{11}$ Department of orthopedics, Yantaishan Hospital, Yantai, China; ${ }^{12}$ Department of Radiology, Peking Union Medical College Hospital, Beijing, China; ${ }^{13}$ Department of Spine Surgery, Beijing Jishuitan Hospital, Beijing, China

Correspondence to: Xiaoguang Cheng. Department of Radiology, Beijing Jishuitan Hospital, Beijing 100035, China. Email: xiao65@263.net; HuiShu Yuan. Department of Radiology, Peking University Third Hospital, Beijing 100191, China. Email: huishuy@sina.com; Jingliang Cheng. Department of MRI, First Affiliated Hospital of Zhengzhou University, Zhengzhou 450052, China. Email: cjr.chj1@vip.163.com; Xisheng Weng. Department of Orthopedics, Peking Union Medical College Hospital, Beijing 100730, China. Email: drwengxsh@163.com; Hao Xu. Department of Nuclear Medicine, First Affiliated Hospital, Jinan University, Guangzhou 510632, China. Email: txh@jnu.edu.cn; Jianbo Gao. Department of Radiology, the First Affiliated Hospital of Zhengzhou University, Zhengzhou 450052, China. Email: cjr.gaojianbo@vip.163.com.

\begin{abstract}
With an aging society, osteoporosis is one of the most common diseases threatening the health of China's elderly population and is an issue that is raising increasing concern. Osteoporosis is characterized by bone loss and increased susceptibility to fragility fractures. Various imaging modalities such as X-ray, CT, MRI and nuclear medicine along with assessment of bone mineral density (BMD) play an important role in its diagnosis and management, and the treatment requires multidisciplinary teamwork. A lack of consensus in the approach to imaging and BMD measurement is hampering the quality of service and patient care in China. Therefore a panel of Chinese experts from the fields of radiology, orthopedics, endocrinology and nuclear medicine reviewed the international guidelines, consensus and literature with the most recent data from China and, taking account of current clinical practice in China, the panel reached this consensus to help guide the diagnosis of osteoporosis using imaging and BMD. This consensus report provides guidelines and standards for the imaging and BMD assessment of osteoporosis and criteria for the diagnosis of osteoporosis in China.
\end{abstract}

Keywords: Osteoporosis; bone mineral density (BMD); imaging; consensus

Submitted Jul 09, 2020. Accepted for publication Jul 28, 2020.

doi: 10.21037/qims-2020-16

View this article at: http://dx.doi.org/10.21037/qims-2020-16

* The member of consensus: Songtao Ai (The Shanghai Ninth People’s Hospital, Shanghai Jiao Tong University School of Medicine), 
Rongjie Bai (Beijing Jishuitan hospital), Xiangran Cai (The First Affiliated Hospital of Jinan University), Xiaodan Chang (The Affiliated Zhongshan Hospital of Dalian University), Jianyu Chen (Sun Yat Sen Memorial Hospital of Sun Yat sen University), Junrong Chen (Sichuan Provincial Orthopedics Hospital), Shuang Chen (Huashan Hospital Affiliated to Fudan University), Wei Chen (Southwest Hospital , Army Medical University), Jingliang Cheng (The First Affiliated Hospital of Zhengzhou University), Xiaoguang Cheng (Beijing Jishuitan Hospital), Jianling Cui (The Third Hospital of Hebei Medical University), Chang Dai (Xinjiang Ale Peoples Hospital), Wei Deng (Beijing Jishuitan Hospital), Shimao Ding (The First Affiliated Hospital of Zhengzhou University), Changwei Ding (Shengjing Hospital of China Medical University), Jianping Ding (The Affiliated Hospital of Hangzhou Normal University), Xia Du (The Affiliated Hospital of Guizhou Medical University), Weihua Feng (The Affiliated Hospital of Qingdao University), Jianbo Gao (The First Affiliated Hospital of Zhengzhou University), Yanzheng Gao (Henan Provincial People's Hospital), Xingjun Gao (Xinyang Central Hospital), Shenchu Gong (Nantong First People's Hospital), Xiangyang Gong (Zhejiang Provincial People's Hospital), Bo He (The First Affiliated Hospital of Kunming Medical University), Yong He (The Second Affiliated Hospital of Guilin Medical University), Guobin Hong (The Fifth Affiliated Hospital Sun Yat-Sen University), Ling Hu (The Editorial Office of Chinese Journal of Radiology), Chen Huang (Yantaishan Hospital), Mingqian Huang (Mount Sinai Hospital, New York), Zhengguo Huang (China-Japan Friendship Hospital), Ning Lang (Peking University Third Hospital), Xinwei Lei (Tianjin First Central Hospital), Chunling Li (Chinese PLA General Hospital), Chunyan Li (The First Affiliated Hospital of Guangxi Medical University), Na Li (Beijing Jishuitan Hospital), Shaolin Li (The Fifth Affiliated Hospital Sun Yat-Sen University), Xiaoming Li (Tongji Hospital, Tongji Medical College, Huazhong University of Science and Technology), Yongli Li (Henan Provincial People's Hospital), Zhenlin Li (West China Hospital of Sichuan University), Qiang Lin (Peking Union Medical College Hospital), Xiangtao Lin (Shandong Provincial Hospital), Hua Liu (Forensic Medical Examination Center of Beijing Public Security Bureau), Li Liu (Forensic Medical Examination Center of Beijing Public Security Bureau), Lin Liu (ChinaJapan Union Hospital of Jilin University), Jihua Liu (The Affiliated Hospital of Qiindao University), Shuxue Liu (Zhongshan Traditional Chinese Medicine Hospital), Xia Liu (Peking University People's Hospital), Yong Lu (Ruijin hospital, Shanghai Jiaotong University School of Medicine), Weifu Lv (Anhui Provincial Hospital), Liheng Ma (The First Affiliated Hospital of Guangdong Pharmaceutical University), Qiang Ma (Beijing Friendship Hospital), Yuanzheng Ma (The 8th Medical Center of Chinese PLA General Hospital), Jinliang Niu (Second Hospital of Shanxi Medical University), Shinong Pan (Shengiing Hospital of China Medical University), Zhanhua Qian (Beijing Jishuitan Hospital), Yongqiang Qian (First Affiliated Hospital of Xi'an Jiaotong University), Cuiping Ren (The First Affiliated Hospital of Zhengzhou University), Yingru Song (Langdong Hospital Affiliated to Guangxi Medical University), Wei Tan (Sichuan Provincial Orthopedics Hospital), Guangyu Tang (Shanghai 10th People's Hospital of Tongji University), Zhanhai Tu (The First Affiliated Hospital of Fujian Medical University), Chenguang Wang (Shanghai Changzheng Hospital), Liang Wang (The 8th Medical Center of Chinese PLA General hospital), Ling Wang (Beijing Jishuitan Hospital), Shaowu Wang (The Second Hospital of Dalian Medical University), Wei Wang (The First Affiliated Hospital of Harbin Medical University), Yan Wang (Chinese PLA General Hospital), Yi-Xiang Wang (The Chinese University of Hong Kong), Zhi Wang (Tianjin Hospital), Yan Wu (The First Affiliated Hospital of Zhengzhou University), Hao Xu (The First Affiliated Hospital of Jinan University), Wenjian Xu (The Affiliated Hospital of Qingdao University), Zhengyang Xu (Chinese PLA General Hospital), Bentao Yang (Beijing Tongren Hospital), Haitao Yang (The First Affiliated Hospital of Chongqing Medical University), Jigang Yang (Beijjng Friendship Hospital), Lian Yang (Xiehe Hospital, Tongji Medical College, Huazhong University of Science and Technology), Minjie Yang (Shenzhen People's Hospital), Weiwu Yao (Shanghai Tongren Hospital, Shanghai Jiaotong University School of Medicine), Qifeng Ying (Zhejiang Provincial People's Hospital), Jinghong Yu (The Second Affiliated Hospital of Inner Mongolia Medical University), Wanjiang Yu (Qingdao Municipal Hospital, East), Huishu Yuan (Peking University Third Hospital), Xianjun Zeng (The First Affiliated Hospital of Nanchang University), Zisan Zeng (The First Affiliated Hospital of Guangxi Medical University), Yunfei Zha (Wuhan University's Renmin Hospital), Zhaohui Zhang (The First Affiliated Hospital, Sun Yat-sen University), Guowei Zhang (Yantaishan Hospital), Lianhe Zhang (Armed Police Zhejiang Province Corps Hangzhou Hospital), Linlin Zhang (The Editorial Office of Chinese Journal of Radiology), Wei Zhang (The Third Hospital of Hebei Medical University), Yan Zhang (Peking Union Medical College Hospital), Heng Zhao (The First Affiliated Hospital of South China University), Quan Zhou (The Third Affiliated Hospital of Southern Medical University), Rong Zhang (Sun Yat-sen University Cancer Center), Xiaodong Zhang (The Third Affiliated Hospital of Southern Medical University), Zhihai Zhang (Aviation General Hospital of China Medical University), Zhuozhao Zheng (Tsinghua University affiliated Beijing Tsinghua Changgung Hospital) and Yuefeng Zou (The First Affiliated Hospital of Nanjing Medical University). 
Osteoporosis is a systemic skeletal disease characterized by bone loss leading to an increased risk of insufficiency fracture $(1,2)$. With China's aging population, there is an increasing attention paid to osteoporosis. A large epidemiological survey using dual-energy X-ray absorptiometry (DXA) amongst people aged fifty and over and standardized for age to data from the 2010 China Census demonstrated that the prevalence in males and females is $6.46 \%$ and $29.13 \%$ respectively (3), meaning that currently there are over 10 million men and 40 million women with osteoporosis in our country $(3,4)$. Insufficiency fracture from osteoporosis is a serious complication that tends to affect the spine, hip, and wrists. It can lead to significant disability and mortality, places a severe burden on society and threatens the health of our elderly population (5-7).

X-ray, CT, MRI, and nuclear medicine studies including DXA and quantitative computed tomography (QCT) measurements of bone mineral density (BMD) are the main methods of diagnosing osteoporosis, predicting fracture risk and evaluating management $(2,8)$. Currently, equipment for measuring BMD are scattered amongst different departments in the Chinese healthcare system with a lack of expert consensus, which negatively affects the advancement of osteoporosis diagnosis and patient care. In order to standardize and improve osteoporosis imaging and BMD measurement, experts from the Bone and Joint Group of the Chinese Society of Radiology, the Chinese Medical Association (CMA), the Musculoskeletal Radiology Society of the Chinese Medical Doctors Association, the Osteoporosis Group of the Chinese Orthopedic Association, and the Bone Density Group of the Chinese Society of Imaging Technology, CMA, reached this "Chinese expert Consensus on the diagnosis of osteoporosis by imaging and bone mineral density", serving as a guideline for radiologists and clinicians for diagnosing and managing osteoporosis. The experts reviewed the related literature, combined with new research in the area and, taking account of medical practice in the Chinese healthcare system, recommend this consensus after thorough discussion.

(I) Osteoporosis definition and classification.

Osteoporosis is a systemic skeletal disease characterized by low bone mass and deterioration of bone microarchitecture with a consequent increase in fragility and susceptibility to insufficiency fracture. It can be classified as primary or secondary. Primary osteoporosis includes postmenopausal osteoporosis (type I), osteoporosis in the elderly (type II), and idiopathic osteoporosis (including in adolescents). Secondary osteoporosis refers to patients with underlying metabolic disease and/or drug induced or from other obvious causes of osteoporosis $(2,8)$. This consensus document mainly applies to the diagnosis of primary osteoporosis.

(II) Risk factors and clinical manifestation of osteoporosis.

Osteoporosis mainly affects post-menopausal women and elderly men. There is no symptom directly from bone loss (9). After an insufficiency fracture, a patient can suffer a variety of symptoms such as pain, loss of height, kyphosis, restricted range of motion, and limited respiration $(2,8,10)$.

Since there are no early symptoms in patients with osteoporosis, individual risk assessment is especially important for patient management. Currently, there are several methods in use. The International Osteoporosis Foundation (IOF) proposed a "oneminute osteoporosis risk assessment" (2) and tools, such as Fracture Risk Assessment (FRAX), which take into account $\mathrm{BMD}$, age, height, weight and other clinical risk factors. The FRAX tool for China can be accessed by logging onto the website: http://www.sheffield.ac.uk/FRAX/toolaspx? country $=2$. However, insufficiency fracture risk assessment must consider the morbidity in the general population and this is unclear for the Chinese population. Thus, there is a limitation to using the FRAX assessment tool in China and we need to establish our own standard.

(III) Diagnostic criteria of osteoporosis.

The principle of diagnosing osteoporosis is to combine clinical history, risk factors, clinical manifestations, imaging findings, BMD measurement and laboratory results together. Osteoporosis can be diagnosed if BMD measurements and clinical manifestations indicate osteoporotic status. Imaging findings and BMD measurements play a critical role here. Once various imaging studies (such as $\mathrm{X}$-ray, CT, MRI, and nuclear medicine studies) demonstrate an insufficiency fracture, osteoporosis can be diagnosed regardless of the findings of a BMD measurement. However, before the occurrence of insufficiency fracture, the diagnosis is mainly based on BMD measurement $(2,12)$. 
It is important to differentiate primary, secondary, or idiopathic osteoporosis, which should be based on age, sex, history, clinical manifestations, laboratory results, and imaging findings. Biochemistry laboratory results can reflect bone formation and bone resorption, aiding classification, differential diagnose and early evaluation of treatment. However, biochemistry cannot be used alone for diagnosing osteoporosis.

(IV) Selection of imaging modalities.

(i) X-ray: an X-ray is the most widely used imaging modality. A plain X-ray can demonstrate decreased bone trabeculae and bone density in osteoporosis. However, these findings are subjective evaluations and not sensitive in the early phases of the disease. If there is bone loss on a plain X-ray, further evaluation with a BMD measurement is warranted. For diagnosis of insufficiency fracture, especially compression fracture of the spine, an X-ray is the first choice and can demonstrate a vertebral deformity. However, if there is subtle vertebral compression, the recommendation is to combine an $\mathrm{X}$-ray with $\mathrm{CT}$ and/or MRI to increase sensitivity.

(ii) CT: a CT scan is also a commonly used imaging modality for the diagnosis of osteoporosis. CT has the advantage of axial plane imaging with the added benefits of multi planar reconstruction (MPR), thus increasing the sensitivity for diagnosing subtle fracture. Additionally, a CT scan is helpful for differentiating osteoporosis from bone tumor and other pathologies.

(iii) MRI: an MRI scan has the advantage of avoiding exposure to ionizing radiation. With high soft tissue contrast, it can show early changes in bone marrow compared to X-ray or CT. Additionally, by detecting marrow edema, it has the advantage of differentiating subtle fracture from bone tumor and infection. However, there is no standardization of the MRI signal, so direct signal measurement is not diagnostic. Some of the special MR sequences can measure fat components in bone marrow, and are useful for osteoporosis evaluation and research, but these sequences are not yet ready for osteoporosis diagnosis. (iv) Nuclear medicine: the nuclear medicine bone scan is not useful for diagnosing primary osteoporosis. However, compression fractures in the spine usually manifest themselves as areas of linear focal uptake. Quantitative bone imaging can be used for monitoring therapy of osteoporosis. Given the specific findings of some of the metabolic bone diseases (such as hyperparathyroidism, Paget's disease, hypertrophic pulmonary osteoarthropathy, and osteomalacia), nuclear medicine bone imaging can be used to diagnose and differentiate causes of secondary osteoporosis. SPECT and SPECT/CT (such as thyroid imaging, parathyroid imaging, and renal function imaging), ${ }^{18} \mathrm{~F}$-FDG PET or PET/ MRI are especially valuable for diagnosing and differentiating causes of secondary osteoporosis.

(V) Diagnosis of fracture related to osteoporosis (insufficiency fracture).

(i) Insufficiency fracture: insufficiency fracture is a serious complication of osteoporosis. The spine is the most frequent site. However, hip insufficiency fracture has the greatest clinical significance. Other common sites are the wrist (Colles' fracture) and proximal humerus (2). Unlike a traumatic fracture, there is usually no history of trauma or only minor trauma (a fall from standing height or even lower counts as minor trauma) for an insufficiency fracture. The diagnosis of insufficiency fracture must fulfill these three criteria: a. no history of trauma or only minor trauma; b. imaging studies demonstrate the fracture; c. exclude the possibility of secondary osteoporosis.

(ii) Diagnosis of insufficiency fracture: spine compression fracture is common in patients with osteoporosis. About three quarters of patients with osteoporotic vertebral fracture do not have a history of trauma or symptoms. A frontal and lateral $\mathrm{X}$-ray examination of the thoracic and lumbar spine is usually the first investigation. Our recommendation is that routine frontal and lateral views of the thoracic and lumbar spine $\mathrm{X}$-ray are performed on anyone over the age of 60 to exclude insufficiency fracture (5). Lateral 
scout image for CT scan and DXA lateral imaging can demonstrate deformity of the vertebral body but cannot demonstrate subtle changes in trabeculae at the vertebral endplate, and thus can only be used as a screening tool. X-ray frontal and lateral views of the chest and abdomen, and especially frontal and lateral views of the spine, can demonstrate compression of the vertebral body. However, without significant awareness of spine compression fracture, it is often missed clinically (3). CT and MRI are best at diagnosing spine fracture and can aid differential diagnosis. Presence of marrow edema of the vertebral body shown on MRI can differentiate acute $v$ s. chronic fracture.

The main manifestation of spine insufficiency fracture is deformity of the vertebral body. Currently, the commonly used criterion for spine insufficiency fracture evaluation is the Genant semi-quantitative (SQ) analysis. It is based on visual assessment of vertebral body height or area decrease and is classified as 0 (normal), grade 1 (mild), grade 2 (moderate), and grade 3 (severe) (14). The Genant SQ method is more suitable for epidemiological studies of spine insufficiency fracture and the evaluation of pharmaceutical therapy (14). Grade 1 and above is generally considered as insufficiency fracture. In clinical practice, visual assessment of change in vertebral body height can cause variations. Wang et al. (15) recommend the evaluation for the presence of endplate and/or cortical fracture (ECF). If there is ECF, it is vertebral body fracture; without ECF it is just vertebral deformity. When there is similar decrease in vertebral body height, vertebral body fracture carries higher risk than vertebral deformity. Severe and multiple vertebral bodies fracture indicate increased future fracture risk.

(iii) Non-spine insufficiency fracture: Patient with non-spine insufficiency fracture usually present with a clear history of trauma. A routine $\mathrm{X}$-ray or CT scan is recommended. Given the decreased bone density in osteoporotic patients and the disadvantage of overlying tissues on a plain X-ray, CT is more sensitive. An MRI scan can be utilized to identify marrow edema using a fat suppression sequence.

(VI) BMD measurement and diagnostic criteria.

The technique of BMD measurement utilizes the principle that there are different degrees of $\mathrm{X}$-ray attenuation when $\mathrm{X}$-rays pass through different media. It is a non-invasive measurement of human bone mineral content, bone density and composition. The current commonly used methods include DXA, QCT and peripheral DXA.

(i) The management of BMD measurement equipment and personnel qualification: the use of X-ray bone density measuring instrument and the regulation of the equipment site and personnel should follow the national regulations on the management of radiation devices. We recommend all BMD measurement practitioners participate in training organized by the Chinese Medical Association, the Chinese Medical Doctor Association, or international societies to ensure a high standard of operation and quality control.

(ii) DXA: DXA uses high and low energy X-rays to scan the human body and measure BMD. Since radiation dose to the patient is extremely low, DXA is the most widely used method of measuring BMD. A DXA result includes measurements of bone mineral content, area and BMD, with BMD the most clinically useful. DXA measures areal bone mineral density (aBMD: units $\mathrm{g} / \mathrm{cm}^{2}$ ). The lumbar spine, hip and forearm are the most common sites for measurement.

The diagnosis of osteoporosis by DXA is based on the T-score, which is calculated in units of the young adult population standard deviation using normal reference data derived from healthy young adults of the same race and sex (2). The reference population should be based on data from the Chinese population, be multi-center, and with a large sample size such as the data from the epidemiological survey conducted by Chinese Center for Disease Control and Prevention, or other similar large normal population study $(2,3,16)$. BMD measurements from different manufacturers' DXA equipment cannot be directly compared without strict quality control and cross 
Table 1 WHO criteria for the diagnosis of osteoporosis using dualenergy X-ray absorptiometry

\begin{tabular}{ll}
\hline Diagnosis & T-score \\
\hline Normal & T-score $\geq-1.0 \mathrm{SD}$ \\
Low bone density & $-2.5 \mathrm{SD}<\mathrm{T}$-score $<-1.0 \mathrm{SD}$ \\
Osteoporosis & T-score $\leq-2.5 \mathrm{SD}$ \\
Severe osteoporosis & $\begin{array}{l}\text { T-score }-2.5 \mathrm{SD} \text { with an insufficiency } \\
\text { fracture }\end{array}$
\end{tabular}

T-score $=$ (measured bone mineral density - peak bone mineral density of normal Chinese young adult)/Standard deviation of peak bone mineral density of normal Chinese young adult (SD).

calibration (17). The criteria for diagnosing osteoporosis using DXA is: scans of the lumbar spine and hip, selecting the L1 to L4 vertebral bodies and the femoral neck and total hip as region of interest (ROI) and using the lowest T-score amongst the 3 ROIs to make the diagnosis. If there is limitation in the measurement of one of the two areas (such as a severe deformity, internal fixation, implants), then the non-dominant forearm should be used as a supplementary area of measurement, using the distal one-third (33\%) radius as the ROI. The DXA diagnostic criteria for osteoporosis (Table 1) are those recommended by an expert panel from the World Health Organization (WHO), and commonly referred to as the 'WHO criteria'. The WHO criteria were established based on data from white women and theoretically are only suitable for use in postmenopausal white women. However, many countries have adopted these criteria in their national osteoporosis diagnosis and treatment guidelines and they have been extended to include elderly men. The academic organization of osteoporosis in China also recommended using this standard in the diagnosis of post-menopausal women and elderly men in China $(2,8)$. The WHO criteria are suitable for use in post-menopausal women and men over the age of 50 . WHO T-scores should never be used to diagnose osteoporosis in children and adolescents. For children, adolescents, pre-menopausal women, and men under the age of 50 , the $Z$-score value is recommended for interpreting $\mathrm{BMD}$ measurements. Z-scores are calculated using BMD reference data derived from healthy subjects of the same age, race and sex. A Z-score $<=-2.0 \mathrm{SD}$ (standard deviation) is defined as "lower than expected range of the same age group" or low bone density $(2,8)$. The normal reference data for children and adolescents should be derived from data for the Chinese population (18). Women with a history of ovariectomy are regarded as equivalent to "post-menopausal" and the T-score can be used for diagnosis.

Because of the planar projection imaging technique of DXA, BMD measurements are influenced by osteoarthritis, scoliosis, osteophytes, vertebral body fracture, vascular calcification and obesity, and these decrease the accuracy of the measurements and may lead to misdiagnosis $(19,20)$. When patients are underweight, overweight or have scoliosis or degenerative changes, QCT is recommended for BMD measurement to minimize their influence or search for evidence of insufficiency fracture.

(iii) QCT: QCT uses data from a clinical CT scan together with calibration data and scan analysis software to measure BMD (21). The CT value of bone tissue in Hounsfield units (HU) varies for different $\mathrm{CT}$ scanners and with $\mathrm{kV}$, so the CT value cannot be used directly as a surrogate of BMD (22). QCT can measure BMD at multiple sites, but is mostly used at the spine or hip. Spine QCT measures the volumetric BMD (vBMD: units $\mathrm{mg} / \mathrm{cm}^{3}$ ) of the central cancellous bone of the vertebral body. Unlike DXA, the result is not influenced by factors such as degenerative changes, scoliosis or body weight (23). For QCT at the hip, a special software application referred to as CTXA generates areal BMD results equivalent to a hip DXA scan $(24,25)$. Radiation dose to the patient from a QCT scan is higher than for DXA, so it is recommended that QCT measurements are made utilizing CT scans acquired for other clinical indications so vBMD can be measured without further radiation exposure. If a CT scan is acquired solely to 
Table 2 Diagnosis of osteoporosis using QCT measurement of lumbar spine bone density

\begin{tabular}{ll}
\hline Diagnosis & Lumbar spine bone density value \\
\hline Normal & Volumetric bone density $>120 \mathrm{mg} / \mathrm{cm}^{3}$ \\
Low bone density & $\begin{array}{l}80 \mathrm{mg} / \mathrm{cm}^{3} \leq \text { volumetric bone density } \\
\leq 120 \mathrm{mg} / \mathrm{cm}^{3}\end{array}$ \\
Osteoporosis & Volumetric bone density $<80 \mathrm{mg} / \mathrm{cm}^{3}$ \\
Severe osteoporosis & $\begin{array}{l}\text { Volumetric bone density }<80 \mathrm{mg} / \mathrm{cm}^{3} \text { with } \\
\text { an insufficiency fracture }\end{array}$
\end{tabular}

", lumbar spine bone mineral density value is the average value of the volumetric bone mineral density measurements obtained from cancellous bone in two lumbar spine vertebral bodies. This standard is suitable for use in post-menopausal women and elderly men. When there is decreased BMD in younger patients, further evaluation should be conducted to exclude secondary causes. When using QCT to diagnose osteoporosis, measuring one area is sufficient and the choice is between spine and hip based on clinical need.

measure $\mathrm{vBMD}$ a low dose technique should be used whenever possible.

In 2007 and 2018 respectively, the International Society for Clinical Densitometry (ISCD) and the American College of Radiology (ACR) proposed diagnostic thresholds for the diagnosis of osteoporosis using lumbar spine QCT $(26,27)$. These thresholds have since been validated and proved to be suitable in the Chinese population (28). In 2018 the Chinese Gerontology and Geriatrics Society Osteoporosis Committee and the Chinese Society of Health Management of the CMA, with other eleven organizations, published the "Guideline on QCT diagnosis of osteoporosis in China (2018)" (29). This standard has since been adopted in the "Guideline for the diagnosis and treatment of elderly osteoporosis in China [2018]" and the "Guideline to the diagnosis and treatment of osteoporosis with integrated traditional Chinese and Western medicine" $(8,30)$. The diagnosis of osteoporosis using lumbar spine QCT is made using the absolute value of the lumbar spine QCT vBMD measurement. It is based on the average of measurements of cancellous $\mathrm{vBMD}$ in two lumbar vertebral bodies (L1 and L2 are most often used). The specific diagnostic criteria are shown in Table 2 (29). There is a high correlation of QCT CTXA and DXA for measuring $\mathrm{BMD}$ at the femoral neck and total hip, with the reported correlation coefficient of $\mathrm{r}=0.81-0.88$ according to the literature $(24,25)$, suggesting that QCT and DXA measurements of hip BMD are equivalent. To interpret CTXA hip QCT measurements, we recommend using the DXA diagnostic standard defining osteoporosis as a T-score $\leq-2.5 \mathrm{SD}$ and hip reference data from a Chinese population (29,31). The current QCT diagnostic standard is based on the Mindways application (Mindways Software, Inc., Austin, TX, USA) with measurements performed at $120 \mathrm{kV}$.

QCT vBMD measurements can be used for monitoring the response to therapy, fracture risk prediction and pre-operative planning for orthopedic surgery. In clinical practice, QCT is mostly obtained simultaneously with a clinical CT scan avoiding additional radiation exposure or scanning time. The Chinese Society of Health Management of the CMA recommends combining QCT with low dose CT screening for lung cancer (29).

(iv) Peripheral BMD measurement: X-ray measurement of BMD in the peripheral skeleton include peripheral QCT (pQCT), peripheral DXA (pDXA) and single energy BMD measurements. The sites measured include the forearm, calcaneus, phalanges, and distal tibia. High-resolution pQCT is used for the evaluation of bone microarchitecture. Since there is less soft tissue around peripheral bone the accuracy and repeatability of the measurements are better. The radiation dose from peripheral BMD measurement equipment is also extremely low, offering better protection for the patient and operator. Quantitative ultrasound can also be used for measuring bone, with the advantage of no radiation exposure. The measurements are related to bone density, but not directly to BMD. Currently, the recommendation is not to use peripheral BMD for diagnosis and evaluation of therapy response in osteoporosis. It can be used only for screening of osteoporosis and 
the fracture risk assessment. Equipment for measuring peripheral BMD has the advantages of being small, easy to transport, low cost, low radiation dose, portable and suitable for osteoporosis screening in smaller hospital and community settings (2).

(VII) New technology and future direction of development.

(i) DXA trabecular bone score (TBS): this is an image texture parameter derived from frontal lumbar spine DXA images and is a new measurement that can provide additional information beyond BMD. In males aged above 50, TBS is a risk factor for hip and other insufficiency fracture. TBS cannot be used alone for determining the start of osteoporosis treatment, but can be combined with other tools such as FRAX for predicting fracture risk (32). There is a need for further research on its clinical application $(33,34)$.

(ii) Spectral CT and dual-energy CT: spectral CT and dual-energy CT (DECT) use double detectors or high and low $\mathrm{kV}$ instant switching or dual source technology, thus enabling differentiation of different tissues (such as calcium, water and fat) $(35,36)$. They can be applied to the quantitative analysis of bone calcium content and BMD measurement. Research has demonstrated high consistency and correlation ( $\mathrm{r}=0.987)$ by using a dual-layer detector CT scanner when comparing BMD measurement results with QCT (36). Spectral $\mathrm{CT}$ and dual-source CT involves a higher radiation dose and therefore it is recommended that BMD measurements with spectral CT are obtained by additional imaging processing of a spectral CT scan performed for other clinical indications, not as a standalone examination for $\mathrm{BMD}$. The clinical value of spectral CT and dual-source CT for BMD measurement in the management of osteoporosis requires further investigation.

(iii) MRI measurement of fat: in addition to the capacity of showing the morphological change of bone with osteoporosis, MRI can be used to evaluate the osteoporosis quantitatively. High resolution MRI can be used to evaluate bone microarchitecture. Single-voxel proton MR spectroscopy and water/fat imaging based on chemical shift can be used to measure the bone marrow proton density fat fraction. Diffusion weighted imaging can evaluate water molecule diffusion in bone marrow. Ultrashort TE (UTE) sequence can evaluate cortical bone (37). Though much research has shown that multiple quantitative parameters obtained by MRI are closely related to BMD, and bone marrow fat content is significantly inversely correlated with $\mathrm{BMD}$, as yet MRI cannot provide a direct measurement of BMD. The clinical research sample sizes in this area are relatively small, and there is a lack of longitudinal studies of the relationship between quantitative parameters obtained by MRI and bone quality. Additionally, MRI evaluation of bone is limited by equipment and technology factors with high cost (38). Currently MRI is not suitable for clinical diagnosis or screening for osteoporosis, but it is used for the evaluation of osteoporosis insufficiency fracture and providing differential diagnosis.

With further development and optimization of MRI equipment and technology, combined with big data and artificial intelligence (AI), MRI quantitative measurement of bone marrow fat and quantitative evaluation of bone cortex using UTE have the potential to provide a new strategy for diagnosis and screening of osteoporosis in the future.

(iv) Nuclear medicine imaging: when PET/CT or SPECT/CT scans are performed on the same integrated scanner they can be used for QCT measurements. QCT calibration needs to be performed before scanning so that $\mathrm{BMD}$ measurements are obtained as part of the routine PET/CT and SPECT/CT imaging. This provides useful additional information for the diagnosis and management of osteoporosis that is further enhanced by the availability of QCT measurements of liver fat content, subcutaneous fat content in the abdomen, organ fat content and muscle content of the extremities.

(v) AI: with the rapid development of computer science technology, there is widespread application of AI. Its application in osteoporosis 
has only just started but is already showing promise (39). Firstly, there may be good value in using AI to detect spine insufficiency fractures due to osteoporosis. Preliminary studies have confirmed the feasibility of using AI technology to study X-ray, CT, MRI, or lateral DXA images for automated detection of vertebral fracture $(40,41)$. Secondly, there are good prospects of using AI for BMD measurement. Preliminary studies have shown that by combining spine CT image with $\mathrm{AI}$ technology it is possible to conduct automated measurements of vertebral body CT values or BMD and fat, muscle area and muscle density $(42,43)$. Lastly, AI can be used for the prediction of fracture risk (44). Given the widespread application of $\mathrm{AI}$ in the diagnosis of osteoporosis, research efforts in this area should be increased.

(vi) Prediction of fracture risk: fracture is a serious complication of osteoporosis, severely limiting patients' quality of life. In clinical practice, to conduct fracture prevention we first need to identify the high-risk population. Reliably predicting fracture risk has always been difficult. Currently, BMD measurement is the main method for diagnosis of osteoporosis and monitoring therapy response. Many studies have shown that fracture risk increases with lower BMD. Using tools such as FRAX we can also use osteoporosis risk factors to predict the risk of future fracture. However, none of these algorithms are ideal, and there is a need for further research to facilitate more accurate predication. China lacks research in long-term follow-up. It is therefore particularly important to improve methods of research in fracture prediction, such as establishing a cohort for long-term follow-up, adopting DXA or QCT BMD measurements, and using integrated analysis of risk factors.

(VIII) Key points of this expert consensus:

(i) For post-menopausal women and elderly men, if there is an osteoporotic fracture identified by imaging, then osteoporosis should be diagnosed regardless of the BMD findings.

(ii) When using DXA to measure lumbar spine and hip, use reference data from the normal
Chinese population. If the T-score at the spine or hip is $\leq-2.5 \mathrm{SD}$, then the diagnosis of osteoporosis can be made.

(iii) When using QCT to measure the lumbar spine, if the volumetric $\mathrm{BMD}<80 \mathrm{mg} / \mathrm{cm}^{3}$, then the diagnosis of osteoporosis can be made. When QCT is used to measure hip using the CTXA application, the results are interpreted the same as a hip DXA scan.

(iv) When BMD measurement meets the criteria for osteoporosis and there is also insufficiency fracture (s), then the diagnosis is severe osteoporosis.

(v) Peripheral BMD measurements can be used for screening for osteoporosis but not for diagnosis.

\section{Acknowledgments}

The authors wish to acknowledge the contribution of professor Zhengyu Jin and Shiyuan Liu of Chinese Society of Radiology (CSR), Professor Zhenlin Zhang of the Chinese Society of Osteoporosis and bone mineral research (CSOBMR), professor Zhenchang Wang of the association of radiologist, the Chinese Medical Doctors Association (CMDA), professor Wei Tian, Yingze Zhang and Qiang Liu of the Chinese Society of Orthopaedic (COA), Professor Haihong $\mathrm{Fu}$ and Zhenlin Li of the Chinese Society of Imaging Technology (CSIT), director LinLin Zhang of the Chinese Journal of Radiology editorial office, director Yongcheng $\mathrm{Hu}$ of Chinese Journal of Orthopaedic editorial office for their support for this consensus.

Funding: The National Key Research and Development Program of China (No. 2020YFC2004900) and Beijing Natural Science Foundation-Haidian Primitive Innovation Joint Fund (grant no. L172019).

\section{Footnote}

Provenance and Peer Review: This article was a free submission to the editorial office, Quantitative Imaging in Medicine and Surgery. The article did not undergo external peer review.

Conflicts of Interest: All authors have completed the ICMJE uniform disclosure form (available at http://dx.doi. org/10.21037/qims-2020-16). YXJW serves as an unpaid Editor-In-Chief of Quantitative Imaging in Medicine and 
Surgery. The other authors have no conflicts of interest to declare.

Open Access Statement: This is an Open Access article distributed in accordance with the Creative Commons Attribution-NonCommercial-NoDerivs 4.0 International License (CC BY-NC-ND 4.0), which permits the noncommercial replication and distribution of the article with the strict proviso that no changes or edits are made and the original work is properly cited (including links to both the formal publication through the relevant DOI and the license). See: https://creativecommons.org/licenses/by-ncnd/4.0/.

\section{References}

1. Kanis JA, Melton LJ 3rd, Christiansen C, Johnston CC, Khaltaev N. The diagnosis of osteoporosis. J Bone Miner Res 1994;9:1137-41.

2. Chinese Society of Osteoporosis and Bone Mineral Research. Guidelines for the diagnosis and treatment of primary osteoporosis (2017). Chinese Journal of Osteoporosis and Bone Mineral Research 2017;10:413-43.

3. Cheng XG, Dong SY, Wang L, Feng J, Sun DM, Zhang Q, Huang JY, Wen QX, Hu R, Li N, Wang QQ, Ma YZ, Fu XX, Zeng Q. Prevalence of osteoporosis in China: a multicenter, large scale survey of a health checkup population. Chinese Journal of Health Management 2019;13:51-8.

4. Zeng Q, Li N, Wang Q, Feng J, Sun D, Zhang Q, Huang J, Wen Q, Hu R, Wang L, Ma Y, Fu X, Dong S, Cheng X. The Prevalence of Osteoporosis in China, a Nationwide, Multicenter DXA Survey. J Bone Miner Res 2019;34:1789-97.

5. Gao C, Xu Y, Li L, Gu WQ, Yi CT, Zhu Q, Gu HA, Chen BH, Wang QQ, Tang F, Xu JL, Hou JM, Song HJ, Wang H, Wang ZL, Zhang ZL. Prevalence of osteoporotic vertebral fracture among community-dwelling elderly in Shanghai. Chin Med J (Engl) 2019;132:1749-51.

6. Xia WB, He SL, Xu L, Liu AM, Jiang Y, Li M, Wang O, Xing XP, Sun Y, Cummings SR. Rapidly increasing rates of hip fracture in Beijing, China. J Bone Miner Res 2012;27:125-9.

7. Tian FM, Zhang L, Zhao HY, Liang CY, Zhang N, Song HP. An increase in the incidence of hip fractures in Tangshan, China. Osteoporos Int 2014;25:1321-5.

8. 2018 China guideline for diagnosis and treatment of senile osteoporosis working group, Ma YZ, Wang YP, Liu Q,
Li CL, Ma X, Wang YJ, Deng LF, He L, Yang NL, Chen BH, Qiu GX, Zhu HM, Tao TZ, Qin L, Wang L, Cheng XG. 2018 China guideline for diagnosis and treatment of senile osteoporosis. Chinese Journal of Osteoporosis 2018;24:1541-67.

9. Kanis JA, Cooper C, Rizzoli R, Reginster JY; Scientific Advisory Board of the European Society for Clinical and Economic Aspects of Osteoporosis (ESCEO) and the Committees of Scientific Advisors and National Societies of the International Osteoporosis Foundation (IOF). European guidance for the diagnosis and management of osteoporosis in postmenopausal women. Osteoporos Int 2019;30:3-44.

10. Zhang ZH, Liu ZH, Li N, Zhang MM, Huang QR, Ma YZ, Wang L, Liu Y, Liu XY, Zhu J, Lan X, Li SC, Yang HB, Yu HF, Tang GY, Zhang W, Yao WW, Li SL, Peng $\mathrm{JH}$, Zhou S, Zhou JS. Expert consensus on the diagnosis of osteoporosis in Chinese Population. Chinese Journal of Osteoporosis 2014:1007-1010.

11. Kanis JA, Johansson H, Harvey NC, McCloskey EV. A brief history of FRAX. Arch Osteoporos 2018;13:118.

12. Kuo TR, Chen CH. Bone biomarker for the clinical assessment of osteoporosis: recent developments and future perspectives. Biomark Res 2017;5:18.

13. Yu W, Yao JP, Lin Q, Mu WB. Cause analysis of missing diagnosis for vertebral fracture on lateral chest radiography. Chinese Journal of Radiology 2010;44:504-7.

14. Genant HK, Wu CY, van Kuijk C, Nevitt MC. Vertebral fracture assessment using a semiquantitative technique. J Bone Miner Res 1993;8:1137-48.

15. Wáng YXJ, Che-Nordin N, Deng M, Leung JCS, Kwok AWL, He LC, Griffith JF, Kwok TCY, Leung PC.

Osteoporotic vertebral deformity with endplate/cortex fracture is associated with higher further vertebral fracture risk: the Ms. OS (Hong Kong) study results. Osteoporos Int 2019;30:897-905.

16. Chinese Society of Osteoporosis and Bone Mineral Research. Epidemiological survey of osteoporosis in China and release of the results of the special action of "healthy bones". Chinese Journal of Osteoporosis and Bone Mineral Research 2019;12:317-8.

17. Jankowski LG, Warner S, Gaither K, Lenchik L, Fan B, Lu Y, Shepherd J. Cross-calibration, Least Significant Change and Quality Assurance in Multiple Dual-Energy X-ray Absorptiometry Scanner Environments: 2019 ISCD Official Position. J Clin Densitom 2019;22:472-83.

18. Xu H, Chen JX, Gong J, Zhang TM, Wu QL, Yuan ZM, Wang JP. Normal reference for bone density in healthy 
Chinese children. J Clin Densitom 2007;10:266-75.

19. Li K, Li XM, Yan D, Cheng KB, Weng L, Li J, Zhang P, Li XY, Gu X, Cheng XG. Diagnostic discordance of osteoporosis using spinal QCT and DXA in Chinese elderly. Chinese Journal of Osteoporosis and Bone Mineral Research 2017;10:271-6.

20. Xu XM, Li N, Li K, Li XY, Zhang P, Xuan YJ, Cheng $\mathrm{XG}$. Discordance in diagnosis of osteoporosis by quantitative computed tomography and dual-energy X-ray absorptiometry in Chinese elderly men. J Orthop Translat 2018;18:59-64.

21. Genant HK, Cann CE, Ettinger B, Gordan GS. Quantitative computed tomography of vertebral spongiosa: a sensitive method for detecting early bone loss after oophorectomy. Ann Intern Med 1982;97:699-705.

22. Engelke K, Lang T, Khosla S, Qin L, Zysset P, Leslie WD, Shepherd JA, Shousboe JT. Clinical Use of Quantitative Computed Tomography-Based Advanced Techniques in the Management of Osteoporosis in Adults: the 2015 ISCD Official Positions-Part III. J Clin Densitom 2015;18:393-407.

23. Link TM. Osteoporosis imaging: state of the art and advanced imaging. Radiology 2012;263:3-17.

24. Cann CE, Adams JE, Brown JK, Brett AD. CTXA hip-an extension of classical DXA measurements using quantitative CT. PLoS One 2014;9:e91904.

25. Cheng X, Wang L, Wang Q, Ma Y, Su Y, Li K. Validation of quantitative computed tomography-derived areal bone mineral density with dual energy X-ray absorptiometry in an elderly Chinese population. Chin Med J (Engl) 2014;127:1445-9.

26. Engelke K, Adams JE, Armbrecht G, Augat P, Bogado CE, Bouxsein ML, Felsenberg D, Ito M, Prevrhal S, Hans DB, Lewiecki EM. Clinical use of quantitative computed tomography and peripheral quantitative computed tomography in the management of osteoporosis in adults: the 2007 ISCD official positions. J Clin Densitom 2008;11:123-62.

27. American College of Radiology. ACR-SPR-SSR practice parameter for the performance of musculoskeletal quantitative computed tomography (QCT). 2018. Available online: https://www.acr.org/-/media/ACR/Files/ Practice-Parameters/qct.pdf?la=en

28. Li K, Chen J, Zhao LF, Chen YZ, Zhou J, Shao JM, Qu HB, Hao XG, Jiang YH, Wu Y, Zhang J, Tang GY, Zhang XQ, Zhang W, Li XR, Wang L, Wang L, Liu SW, Wu J, Ma YZ, Cheng XG.The establishment of QCT spinal $v B M D$ reference database and the validation of the diagnosis criteria of osteoporosis with QCT for Chinese. Chinese Journal of Osteoporosis 2019;25:1257-62,1272.

29. The China guideline for the diagnosis criteria of osteoporosis with quantitative computed tomography (QCT) working group, Cheng XG, Wang L, Zeng Q, $\mathrm{Wu} \mathrm{J}$. The China guideline for the diagnosis criteria of osteoporosis with quantitative computed tomography (QCT) (2018). Chinese Journal of Osteoporosis 2019;25:733-7.

30. Professional Committee of orthopedics and traumatology of China Association of Integrated Traditional Chinese and Western Medicine. Guide to diagnosis and treatment of osteoporosis with integrated traditional Chinese and western medicine. National Medical Journal of China 2019;99:3524-33.

31. Compston J, Cooper A, Cooper C, Gittoes N, Gregson C, Harvey N, Hope S, Kanis JA, McCloskey EV, Poole KES, Reid DM, Selby P, Thompson F, Thurston A, Vine $\mathrm{N}$; National Osteoporosis Guideline Group (NOGG). UK clinical guideline for the prevention and treatment of osteoporosis. Arch Osteoporos 2017;12:43.

32. The international society for clinical densitometry (ISCD). 2019 ISCD Official Positions - Adult. 2019 May 28. Available online: https://www.iscd.org/officialpositions/2019-iscd-official-positions-adult/

33. Silva BC, Leslie WD, Resch H, Lamy O, Lesnyak O, Binkley N, McCloskey EV, Kanis JA, Bilezikian JP. Trabecular bone score: a noninvasive analytical method based upon the DXA image. J Bone Miner Res 2014;29:518-30.

34. Martineau P, Leslie WD. Trabecular bone score (TBS): Method and applications. Bone 2017;104:66-72.

35. Chen M, Zhang Y, Yuan HS. A preliminary study of the correlation and agreement of bone mineral density measurements between rapid-kilovoltage-switching dualenergy CT and QCT. Chinese Journal of Osteoporosis 2019;25:326-9,370.

36. Mei K, Schwaiger BJ, Kopp FK, Ehn S, Gersing AS, Kirschke JS, Muenzel D, Fingerle AA, Rummeny EJ, Pfeiffer F, Baum T, Noël PB. Bone mineral density measurements in vertebral specimens and phantoms using dual-layer spectral computed tomography. Sci Rep 2017;7:17519.

37. Guerri S, Mercatelli D, Aparisi Gómez MP, Napoli A, Battista G, Guglielmi G, Bazzocchi A. Quantitative imaging techniques for the assessment of osteoporosis and sarcopenia. Quant Imaging Med Surg 2018;8:60-85.

38. Adams JE. Advances in bone imaging for osteoporosis. Nat 
Rev Endocrinol 2013;9:28-42.

39. Burns JE, Yao J, Summers RM. Artificial intelligence in musculoskeletal imaging: a paradigm shift. J Bone Miner Res 2020;3 5:28-35.

40. Burns JE, Yao J, Summers RM. Vertebral body compression fractures and bone density: automated detection and classification on CT images. Radiology 2017;284:788-97.

41. Cruz AS, Lins HC, Medeiros RVA, Filho JMF, da Silva SG. Artificial intelligence on the identification of risk groups for osteoporosis, a general review. Biomed Eng Online 2018;17:12.

42. Graffy PM, Liu J, Pickhardt PJ, Burns JE, Yao J, Summers RM. Deep learning-based muscle segmentation

Cite this article as: Cheng $\mathrm{X}$, Yuan $\mathrm{H}$, Cheng $\mathrm{J}$, Weng $\mathrm{X}$, Xu H, Gao J, Huang M, Wáng YXJ, Wu Y, Xu W, Liu L, Liu H, Huang C, Jin Z, Tian W; on behalf of Bone and Joint Group of Chinese Society of Radiology, Chinese Medical Association (CMA), Musculoskeletal Radiology Society of Chinese Medical Doctors Association, Osteoporosis Group of Chinese Orthopedic Association, Bone Density Group of Chinese Society of Imaging Technology, CMA. Chinese expert consensus on the diagnosis of osteoporosis by imaging and bone mineral density. Quant Imaging Med Surg 2020;10(10):20662077. doi: 10.21037/qims-2020-16 and quantification at abdominal CT: application to a longitudinal adult screening cohort for sarcopenia assessment. Br J Radiol 2019;92:20190327.

43. Jang S, Graffy PM, Ziemlewicz TJ, Lee SJ, Summers RM, Pickhardt PJ. Opportunistic Osteoporosis Screening at Routine Abdominal and Thoracic CT: Normative L1 Trabecular Attenuation Values in More than 20000 Adults. Radiology 2019;291:360-7.

44. Ferizi U, Besser H, Hysi P, Jacobs J, Rajapakse CS, Chen C, Saha PK, Honig S, Chang G. Artificial Intelligence Applied to Osteoporosis: A Performance Comparison of Machine Learning Algorithms in Predicting Fragility Fractures From MRI Data. J Magn Reson Imaging 2019;49:1029-38. 